\title{
Adjunctive Perampanel in Older Patients With Epilepsy: A Multicenter Study of Clinical Practice
}

\author{
Simona Lattanzi ${ }^{1}$ (1) Claudia Cagnetti ${ }^{1} \cdot$ Nicoletta Foschi $^{1} \cdot$ Roberta Ciuffini $^{2} \cdot$ Elisa Osanni ${ }^{3} \cdot$ Valentina Chiesa $^{4}$. \\ Filippo Dainese $^{5} \cdot$ Fedele Dono $^{6} \cdot$ Maria Paola Canevini $^{4,7} \cdot$ Giacomo Evangelista $^{6}$. Francesco Paladin ${ }^{5}$. \\ Emanuele Bartolini ${ }^{8} \cdot$ Federica Ranzato $^{9} \cdot$ Annacarmen Nilo $^{10}$. Giada Pauletto ${ }^{11}$. Daniela Marino ${ }^{12}$. \\ Eleonora Rosati ${ }^{13} \cdot$ Paolo Bonanni $^{3} \cdot$ Alfonso Marrelli $^{14}$
}

Accepted: 16 May 2021 / Published online: 2 June 2021

(c) The Author(s) 2021

\begin{abstract}
Background Clinical data regarding use of newer antiseizure medications (ASMs) in an older population are limited. In randomized-controlled, placebo-controlled trials, older patients are under-represented, and protocols deviate markedly from routine clinical practice, limiting the external validity of results. Studies performed in a naturalistic setting are a useful complement to characterize the drug profile. Perampanel is a third-generation ASM and the first and only non-competitive alfa-amino-3-hydroxyl-5-methyl-4-isoxazole-propionate receptor antagonist.

Objective The aim of this study was to assess the effectiveness and tolerability of adjunctive perampanel over a 1-year period in a population of older patients with epilepsy treated in a real-world setting.

Methods Older ( $\geq 65$ years of age) patients prescribed add-on perampanel at 12 Italian epilepsy centers were retrospectively identified. Seizure occurrence, adverse events (AEs), and drug withdrawal were analyzed. Effectiveness outcomes included the rates of seizure response ( $\geq 50 \%$ reduction in baseline monthly seizure frequency), seizure freedom, and treatment discontinuation. Safety and tolerability outcomes were the rate of treatment discontinuation due to AEs and the incidence of AEs. Results A total of 92 patients with a median age of 69 (range 65-88) years were included. The median daily dose of perampanel at 12 months was $6 \mathrm{mg}$ (interquartile range 4-6 mg). At 12 months, $53(57.6 \%)$ patients were seizure responders, and 22 (23.9\%) patients were seizure free. Twenty (21.7\%) patients discontinued perampanel; the reasons for treatment withdrawal were insufficient efficacy $(n=6 / 20 ; 30.0 \%)$, AEs $(n=12 / 20 ; 60.0 \%)$, and a combination of both $(n=2 / 20 ; 10 \%)$. The most common AEs included irritability (8.7\%), somnolence (4.3\%), and dizziness/vertigo (4.3\%). The rate of behavioral and psychiatric AEs was higher in patients with history of psychiatric comorbidities $(p=0.044)$. There were no differences in the occurrence of behavioral and psychiatric AEs according to the concomitant use of levetiracetam $(p=0.776)$ and history of cognitive decline ( $p=0.332)$.

Conclusions Adjunctive perampanel was associated with improvement in seizure control and good tolerability in a real-life setting and can represent a viable therapeutic option in older patients with epilepsy.
\end{abstract}

Simona Lattanzi

alfierelattanzisimona@gmail.com

Extended author information available on the last page of the article 


\section{Key Points}

Clinical data regarding use of newer antiseizure medications (ASMs) in an older population are limited.

Perampanel is a third-generation ASM and the first and only non-competitive alfa-amino-3-hydroxyl-5-methyl4-isoxazole-propionate receptor antagonist.

Adjunctive perampanel was associated with improvement in seizure control and good tolerability in a real-life setting.

Perampanel can represent a viable therapeutic option in older patients with epilepsy.

\section{Introduction}

Epilepsy has a peak incidence in older age groups, with an annual incidence of 134 per 100,000 in people aged $\geq 65$ years and a prevalence rate of approximately $1.5 \%$ by age 75 years $[1,2]$. With the aging population, treatment of epilepsy in older patients has become part of daily practice and this subgroup of vulnerable patients is rapidly growing [3].

The management of epilepsy in an older adult is challenging. Age-related physiological changes, including decrease in renal excretion, impairment of hepatic function, and lower protein binding due to reduced albumin levels can result in changes of pharmacokinetics [3]. The pharmacodynamic properties of antiseizure medications (ASMs) can also be affected by variations in receptor density and sensitivity [4]. Furthermore, the rates of comorbidities and comedications are high and polypharmacy raises the risk for drug-drug interactions, drug toxicity, and poor medication adherence [5]. Due to the long-term metabolic derangements, increase of vascular risk factors, and the high potential to affect the metabolism of drugs commonly prescribed in older adults such as oral anticoagulants, antiarrhythmics, statins, and antihypertensive agents, first-generation ASMs are preferably avoided $[3,6,7]$. The assessment of the efficacy and tolerability of the newer ASMs in older adults is, hence, of paramount clinical relevance.

Perampanel is the first and only non-competitive alphaamino-3-hydroxyl-5-methyl-4-isoxazole-propionate (AMPA) receptor antagonist specifically engineered to block glutamate activity at postsynaptic AMPA receptors [8]. It is a third-generation ASM and has been licensed as adjunctive treatment of focal seizures in patients aged $\geq 4$ years and as adjunctive treatment of primary generalized tonic-clonic seizures associated with idiopathic generalized epilepsy in patients aged $\geq 12$ years ( $\geq 7$ years in the EU) based on the results from randomized controlled trials (RCTs). In the USA, the drug has also received marketing authorization as monotherapy.

The aim of this study was to assess the effectiveness and tolerability of adjunctive perampanel over a 1-year period in a population of older patients with epileps treated in the context of real-world clinical practice.

\section{Methods}

\subsection{Participants}

Retrospectively identified study participants were older ( $\geq 65$ years of age) patients regularly attending 12 Italian epilepsy centers who were prescribed add-on perampanel (January 2014-January 2020), were on stable treatment with one or more ASM during the prior 90 days, and had at least 12 months of follow-up.

Data on demographics, clinical history, type of seizures and epilepsy [9], etiology, previous/concomitant ASMs, and baseline seizure frequency (monthly seizure frequency during the 3 months before starting perampanel) were collected.

Concomitant ASMs were classified as enzyme-inducing ASMs (EiASMs; carbamazepine, phenytoin, phenobarbital, primidone) and non-EiASMs (any other ASM); patients were described as taking EiASMs if they were taking at least one EiASM during perampanel treatment.

Seizure occurrence, adverse events (AEs), and drug withdrawal were retrieved from patient seizures diaries and clinical records of 3-, 6-, and 12-month follow-up visits, as standard practice when a new ASM is initiated. Exclusion criteria were history of alcoholism, drug abuse, conversion disorders, or other non-epileptic ictal events.

\subsection{Outcomes}

Effectiveness outcomes included the rates of seizure response $(\geq 50 \%$ reduction in baseline monthly seizure frequency), seizure freedom, seizure worsening ( $>25 \%$ increase in monthly seizure frequency relative to baseline), and treatment discontinuation at 12 months.

Further analyses were performed using data obtained from the visits at 3 and 6 months. Seizure freedom at each time point was defined as the occurrence of no seizures since at least the previous visit: at 12 months, it was considered as no seizures during the preceding 6 months, and at 3 and 6 months it was defined as lack of seizures since baseline or the 3-month visit, respectively. 
Seizure-free patients were included in the seizure response group unless they were already free from seizures during the baseline period.

Safety and tolerability outcomes included the rate of treatment discontinuation due to AEs and the incidence of AEs considered perampanel-related by participating physicians.

\subsection{Statistical Analysis}

Values were presented as mean $( \pm \mathrm{SD})$ or median (interquartile range) for continuous variables and number (percent) of subjects for categorical variables. Comparisons were made using the Mann-Whitney test or Chi-squared test, as appropriate.

Exploratory analyses were performed to evaluate the impact of concomitant use of EiASMs, concomitant use of levetiracetam, presence of psychiatric comorbidities, and cognitive impairment on study outcomes.

Results were considered significant for $p$ values $<0.05$ (two sided). Data analysis was performed using STATA/IC 13.1 (StataCorp LP, TX, USA).

\section{Results}

Ninety-two patients were included in the study. The median age of the patients was 69 (66-73) years, ranging from 65 to 88 years, and $46(50.0 \%)$ were males. Patients had a history of a median of 4 (2-7) lifetime ASMs and perampanel was added to a median of $2(1-2)$ concomitant ASMs. The reason for starting perampanel was inadequate seizure control in $76(82.6 \%)$ patients, presence of AEs in $4(4.3 \%)$ patients and both reasons in $12(13.0 \%)$ patients. The baseline monthly seizure frequency was $2(1-6)$, and $3(3.3 \%)$ patients were free from seizure when perampanel was added. Baseline characteristics of participants are summarized in Table 1. Perampanel was introduced because of inadequate seizure control in 76 (82.6\%) patients, AEs of other ASMs in 4 (4.4\%) and a combination of both reasons in $12(13.0 \%)$ patients.

The median daily dose of perampanel at 3 months was 4 (4-4) $\mathrm{mg}$, at 6 months it was 5 (4-6) $\mathrm{mg}$ and at 12 months it was 6 (4-6) mg. At 12 months, the proportions of patients prescribed to daily perampanel dosages of $2 \mathrm{mg}, 4 \mathrm{mg}$, $6 \mathrm{mg}, 8 \mathrm{mg}, 10 \mathrm{mg}$, and $12 \mathrm{mg}$ were $4.0 \%, 36.0 \%, 41.3 \%$, $13.3 \%, 4.0 \%$, and $1.3 \%$, respectively. Titration at 3 months was $2 \mathrm{mg} /$ week in 15 patients (16.3\%), 2 mg every 2 weeks in 63 patients $(68.5 \%)$, and $2 \mathrm{mg}$ every $3-4$ weeks in 14 patients (15.2).

At 12 months, 53 (57.6\%) patients had their seizure frequency reduced by $50 \%$ or more in comparison with baseline and $22(23.9 \%)$ patients were seizure free. The response and seizure freedom rates at 3 and 6 months
Table 1 Baseline characteristics of patients

\begin{tabular}{|c|c|}
\hline Characteristics & All patients $(n=92)$ \\
\hline Age, years & $69(66-73)$ \\
\hline Male sex & $46(50.0)$ \\
\hline Age at epilepsy onset, years & $48(23-64)$ \\
\hline Duration of epilepsy, years & $22(7-49)$ \\
\hline \multicolumn{2}{|l|}{ Type of seizures } \\
\hline Focal onset & $73(79.3)$ \\
\hline Focal to bilateral tonic-clonic & $22(23.9)$ \\
\hline Generalized onset & $5(5.4)$ \\
\hline \multicolumn{2}{|l|}{ Etiology } \\
\hline Structural & $58(63.0)$ \\
\hline Immune & $2(2.2)$ \\
\hline Infectious & $4(4.3)$ \\
\hline Unknown & $28(30.4)$ \\
\hline \multicolumn{2}{|l|}{ Medical history } \\
\hline Hypertension & $43(46.7)$ \\
\hline Dyslipidemia & $32(34.8)$ \\
\hline Diabetes mellitus & $8(8.7)$ \\
\hline Stroke & $10(10.9)$ \\
\hline Coronary heart disease & $9(9.8)$ \\
\hline Learning disability & $11(12.0)$ \\
\hline Psychiatric comorbidity & $43(46.7)$ \\
\hline Mild cognitive impairment/dementia & $30(32.6)$ \\
\hline Number of previous ASMs & $4(2-7)$ \\
\hline Number of concomitant ASMs & $2(1-2)$ \\
\hline Concomitant EiASMs & $31(33.7)$ \\
\hline Concomitant sodium-channel blockers & $60(65.2)$ \\
\hline Baseline monthly seizure frequency* & $2(1-6)$ \\
\hline
\end{tabular}

Data are median (IQR) for continuous variables, and $n(\%)$ for categorical variables

ASMs anti-seizure medication, EiASMs enzyme-inducing ASMs, IQR interquartile range

*Based on the number of seizures during the 90 days before starting adjunctive perampanel

are shown in Fig. 1. During the 12-month follow-up, 22 (23.9\%) patients had a reduction in the dosage of one or more concomitant ASMs, and withdrawal of one or more concomitant ASMs occurred in 30 (32.6\%) patients. At 12 months, there was a numerically higher number of responders to perampanel in patients on concomitant nonEiASMs versus patients on EiASMs $(80.0 \%$ vs $52.0 \%$; $p=0.012$ ) and more patients taking non-EiASMs were free from seizures than patients on EiASMs $(36.0 \%$ vs $16.0 \% ; p=0.073)$.

During the 1-year study period, 20 (21.7\%) patients of the cohort discontinued perampanel. The reasons of perampanel withdrawal were insufficient efficacy $(n=6 / 20 ; 30.0 \%)$, AEs $(n=12 / 20 ; 60.0 \%)$, and a combination of both $(n=2 / 20$; $10 \%)$. Out of 20 patients who withdrew perampanel, 10 


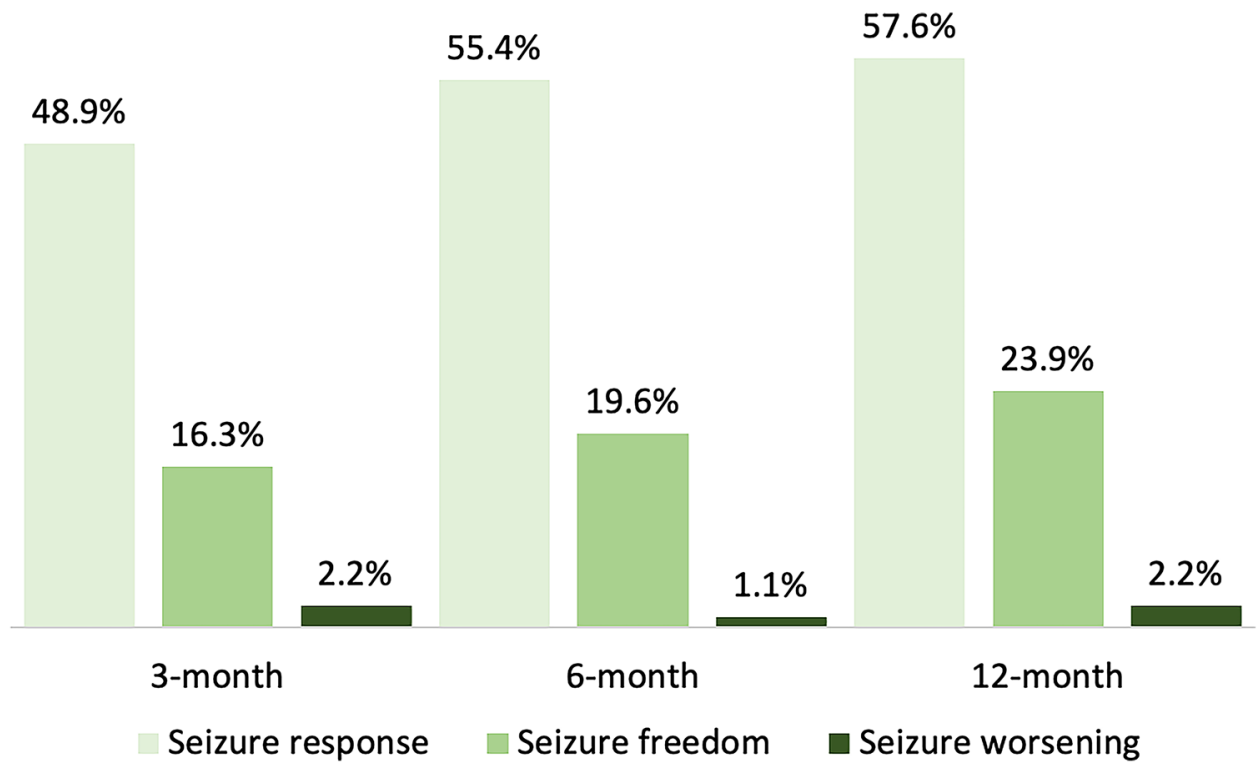

Fig. 1 Clinical response to adjunctive perampanel. Rates of seizure response, seizure freedom, and seizure worsening at 3, 6, and 12 months are reported. Percentages are estimated on the full study population $(n=92)$. Seizure response was defined as a reduction in seizure frequency $\geq 50 \%$ in comparison with baseline seizure frequency. Seizure freedom at each time point was defined as the occurrence of

Table 2 Adverse events with adjunctive perampanel treatment

Most frequently reported adverse events (reported by $\geq 2 \%$ of patients)

\begin{tabular}{ll}
\hline Irritability, $n(\%)$ & $8(8.7)$ \\
Somnolence, $n(\%)$ & $4(4.3)$ \\
Dizziness/vertigo, $n(\%)$ & $4(4.3)$ \\
Instability/ataxia, $n(\%)$ & $3(3.3)$ \\
Insomnia, $n(\%)$ & $3(3.3)$ \\
Fatigue, $n(\%)$ & $2(2.2)$ \\
Mood change, $n(\%)$ & $2(2.2)$ \\
Anxiety, $n(\%)$ & $2(2.2)$ \\
Aggressiveness, $n(\%)$ & $2(2.2)$ \\
\hline
\end{tabular}

AEs reported by $<2 \%$ of patients: mental confusion/slowing/psychomotor retardation, nausea, tachycardia, urinary urgency, and weight gain (all $n=1,1.1 \%)$

$(50.0 \%)$ had their treatment discontinued at 3 months, 3 $(15.0 \%)$ at 6 months and $7(35.0 \%)$ at 12 months.

At least one AE was reported by $32(34.8 \%)$ patients. The AEs were rated in intensity as mild in $65.7 \%$ and moderate in $34.3 \%$ cases; no severe AEs were reported. The most common AEs observed in the study cohort included irritability (8.7\%), somnolence (4.3\%), dizziness/vertigo (4.3\%), instability/ataxia (3.3\%), and insomnia (3.3\%) (Table 2).

The rate of patients experiencing behavioral and psychiatric AEs was significantly higher in patients with a history no seizures since at least the previous visit. Seizure worsening was defined as an increase in seizure frequency $>25 \%$ in comparison with baseline seizure frequency. Seizure-free patients were included in the seizure response group unless they were already free from seizures during the baseline period

of psychiatric comorbidities than in those without $(23.3 \%$ vs $8.2 \% ; p=0.044)$. There were no differences in the occurrence of behavioral and psychiatric AEs according to the concomitant use of levetiracetam $(p=0.776)$ and the history of cognitive decline, either mild cognitive impairment or dementia $(p=0.332)$.

\section{Discussion}

In a retrospective analysis of older patients with predominantly focal onset seizures treated with adjunctive perampanel in a real-world setting, about $80 \%$ of the patients were still on treatment at 12 months, $60 \%$ achieved a reduction in their seizure frequency by $50 \%$ or greater, and one quarter of the population was free from seizures. Further, the reduction in dosage or discontinuation of one or more concomitant ASMs occurred in half of the study cohort. The most common dosage of perampanel at 12 months from starting treatment was $6 \mathrm{mg} /$ day and titration generally occurred slowly with dose increment every $\geq 2$ weeks. Adverse events were reported during the 1-year follow-up by $35 \%$ of the patients and ranged from mild to moderate intensity; the most commonly observed were irritability, somnolence, and dizziness.

Clinical data regarding use of newer ASMs in an older population are limited and obtaining more information is essential. In regulatory epilepsy trials, older patients are often under-represented as comorbidities or physical frailty 
may interfere with recruitment and participation and affect outcomes [10]. Further, randomized-controlled protocols deviate markedly from routine clinical practice and the restrictive eligibility criteria, rigid titration schedules, little or no flexibility in dosing schemes, and short follow-up duration limit the external validity of the results [11, 12].

The sub-analysis of the three randomized, double-blind, placebo-controlled, phase III trials in patients with focal seizures provided data for only 20 patients aged $\geq 65$ years randomized to add-on perampanel, which comprised $1.9 \%$ of the total pooled population [13]. A reduction $\geq 50 \%$ in monthly seizure frequency was achieved by $22 \%$ and $43 \%$ in the 8- and 12-mg daily groups; only three and one patients received perampanel at the dose of 2 and $4 \mathrm{mg} /$ day, respectively. The rate of treatment discontinuation due to poor tolerability was $20 \%$, and all patients were in the $12-\mathrm{mg}$ group. Treatment-emergent AEs were reported by $85 \%$ of the elderly cohort, and the most common included dizziness, somnolence, irritability, gait disturbance, falls, and balance disorder [13]. Of note, the registration trials mostly focused on drug-resistant patients with higher baseline seizure frequency, making a comparison with the present study difficult.

Studies performed in a naturalistic setting are needed as a useful complement to fully characterize the clinical profile of ASMs in this special population and under the usual circumstances of healthcare practice. To date, few real-world studies based on small sample sizes are available about the use of perampanel in older patients.

A pooled, individual-level analysis of observational studies on perampanel treatment in routine clinical practice on 2396 patients from 45 sites in Europe included 135 patients aged $\geq 65$ years [14]. At 12 months, the median perampanel daily dose was $6 \mathrm{mg}$ and the retention rate was $47.8 \%$ with intolerability accounting for nearly half of all cases of drug withdrawal. Data on seizure outcome were available for only 46 patients, and seizure freedom for at least 6 months was achieved by $28.3 \%$ of the cases. The pragmatic seizure freedom rate estimated on the full analysis set was $9.7 \%$. Adverse events were reported by nearly $80 \%$ of patients, and dizziness (24.7\%), somnolence (16.5\%), and behavioral disturbances (16.5\%) were the most common [14]. In a retrospective, double-center study comparing the effectiveness of add-on therapy with perampanel and brivaracetam, ten patients treated with perampanel were aged $\geq 62$ years; at 12 months, $20 \%$ of patients were shown to be seizure responders and $40 \%$ were free from seizures [15]. Twenty older patients with a mean age of 70 years were followed in a single-center prospective audit; over 57 months, $75 \%$ of patients were still taking perampanel and $35 \%$ were seizure free [16]. Thirty-five percent of patients experienced AEs, and fatigue (20\%) and vertigo (15\%) were the most frequent [16]. Importantly, the heterogeneity in study design, number of centers involved, characteristics and numbers of included patients, and the discrepancies in length of follow-up, and definition and assessment of outcomes do not allow direct comparisons between the available studies.

A retrospective chart review of medical records from five European hospitals aimed to evaluate the use of perampanel in adult patients with status epilepticus (SE). In a cohort of 1319 patients experiencing SE, 52 (3.9\%) received perampanel [17]. The mean age of the population was 60.5 years, with a range from 19 to 91 years. The median initial perampanel dose was $6 \mathrm{mg} /$ day (range 2-24), which was up-titrated to a median maximum dose of $10 \mathrm{mg} /$ day (range 4-24). Perampanel was the last drug added in $61.5 \%$ of patients, with response attributed to perampanel in $36.5 \%$ of cases. The most common AEs during perampanel administration were dizziness and somnolence, which occurred at a rate of $1.9 \%$, and no serious adverse effects were documented [17]. These data highlight the potential of perampanel in the acute care setting of SE management and suggest that high doses of perampanel can be safely used in older patients $[17,18]$.

Older patients taking perampanel and concomitant nonEiASMs had a greater clinical response than patients not taking concomitant EiASMs. This observation is consistent with data observed in adults in both RCTs [19] and realworld studies [20]. Given the plasma concentration-effect relationship for perampanel and its extensive metabolism via the cytochrome P450 (CYP) isoenzyme system [21], the concomitant administration of CYP3A4 inducers can increase oral clearance, decrease systemic exposure, and ultimately reduce clinical efficacy. The recognition of these pharmacokinetic interactions is important in the optimization of dose when perampanel is added to enzyme inducers or when concomitant medications with enzyme-inducing properties are withdrawn.

Irritability was the most common $\mathrm{AE}$ observed in the study cohort and the likelihood to develop a psychiatric $\mathrm{AE}$ was higher among older patients presenting with psychiatric comorbidities at baseline. These findings expand the experience already accumulated in adult cohorts of patients [20], support the potential role of glutamate in psychiatric issues [22], and highlight the influence of the individual clinical profile on the propensity to develop psychiatric AEs during treatment with ASMs [23]. Patients and caregivers should be counseled regarding the potential risk of behavioral events with perampanel and increased caution is needed when treating older patients already presenting with psychiatric issues. Of note, we did not find an increased risk of psychiatric AEs when perampanel was added to levetiracetam, reinforcing the evidence that concomitant treatment with levetiracetam has no appreciable effect on the occurrence of these kinds of AEs in patients receiving perampanel [24]. Although the study did not consider measures specifically aimed to assess the influence of treatment on neuropsychological functions, 
no cognitive complaints emerged. Inasmuch as specific data on the cognitive impact of perampanel treatment in older patients are not available, this preliminary evidence suggests the good cognitive profile demonstrated in the younger population in older adults also $[25,26]$.

The elevated risk of dizziness, balance disorders, and falls reported in the pooled analysis of phase III studies of adjunctive perampanel [13] was not found in this real-world analysis. The high rate of stroke etiology, which is itself a significant risk for falls [27], the higher dosing, and the faster titration that characterized the RCTs may contribute to explain such discrepancies. Finally, there was no difference in the overall tolerability profile of add-on perampanel between patients with cognitive impairment, which represented around one third of the whole study cohort, and cognitively healthy individuals. Given the high prevalence of cognitive dysfunction in older patients and the relationship between seizures and dementia [28], the availability of a well-tolerated ASM in this population may be of clinical relevance.

\subsection{Strengths and Limitations}

This study provides novel insights into the effectiveness of adjunctive perampanel in older patients. Major strengths include the recruitment at multiple sites, the real-world setting, which allowed the generalizability of the findings to everyday clinical practice, and the analysis of tolerability profile by the history of cognitive decline. Although only few patients were recruited across the years, highlighting the challenges to obtain data in an older population with epilepsy, the sample size of this study is larger than the cohorts included in any of the currently available studies to assess the efficacy of perampanel in the elderly. Different shortcomings need to be considered in the interpretation of results, like the open-label and retrospective design, which might have introduced potential sources of biases, and the unavailability of data on seizure frequency by seizure subtypes and on plasma concentrations of ASMs. Importantly, the lack of a control group of matching patients being treated with an alternative drug does not allow us to draw conclusions about the comparative efficacy and safety of perampanel and other ASMs, and direct comparison to other studies can be hampered by the heterogeneity in epilepsy types, etiologies, prior and concomitant exposure to ASMs, and seizure frequency. Few patients were titrated to $10-12 \mathrm{mg} /$ day, limiting most of the evidence to the lower dosages, and the lack of information about the proportion of patients in which high dosages could not be achieved due to AEs did not allow a comprehensive overview of the tolerability profile of perampanel. Additionally, the collection of AEs and comorbidities from clinical records of follow-up visits in absence of standardized questionnaires and global comorbidity indices like the Charlson Comorbidity Index might have resulted in possible underreporting and not a fully complete overview of the population.

\section{Conclusion}

Adjunctive perampanel is overall associated with the improvement in seizure control and good tolerability and can be a viable therapeutic option in older patients with epilepsy. Further research considering patient-reported outcomes, health-related quality of life, neuropsychological tests, and inventories is needed to fully explore the potential of perampanel treatment in the older population and provide more information for clinical decisions.

\section{Declarations}

Funding Open access funding provided by Università Politecnica delle Marche within the CRUI-CARE Agreement. No funding has been received for the conduct of this study.

Conflict of interest Simona Lattanzi has received speaker's or consultancy fees from Eisai, GW Pharmaceuticals, and UCB Pharma and has served on advisory boards for Angelini, Arvelle Therapeutics, BIAL, and GW Pharmaceuticals. Valentina Chiesa has received speaker's or consultancy fees from Eisai, UCB Pharma, and Livanova. Fedele Dono has received speaker's fees from Eisai. Maria Paola Canevini has received speaker's or consultancy fees from Bial, EISAI, Italfarmaco, Sanofi, and UCB. All other authors have nothing to disclose.

Ethics approval The study was approved by the ethical committee at any participating center and conducted in accordance with the Declaration of Helsinki.

Consent to participate Informed consent was obtained from every patient or legal representative.

Consent for publication Not applicable.

Data availability Anonymized data will be shared by request from any qualified investigator.

Code availability Not applicable.

Author contributions SL drafted the concept, analyzed data and wrote the manuscript. CC, NF, CR, EO, VC, FD, FD, MPC, GE, FP, EB, FR, AN, GP, DM, ER, PB, and AM collected data. All authors critically revised the work and contributed to the final manuscript. All authors approved the final manuscript for submission.

Open Access This article is licensed under a Creative Commons Attribution-NonCommercial 4.0 International License, which permits any non-commercial use, sharing, adaptation, distribution and reproduction in any medium or format, as long as you give appropriate credit to the original author(s) and the source, provide a link to the Creative Commons licence, and indicate if changes were made. The images or other third party material in this article are included in the article's Creative Commons licence, unless indicated otherwise in a credit line to the 
material. If material is not included in the article's Creative Commons licence and your intended use is not permitted by statutory regulation or exceeds the permitted use, you will need to obtain permission directly from the copyright holder. To view a copy of this licence, visit http://creativecommons.org/licenses/by-nc/4.0/.

\section{References}

1. Hauser WA, Annegers JF, Kurland LT. Incidence of epilepsy and unprovoked seizures in Rochester, Minnesota: 1935-1984. Epilepsia. 1993;34:453-68.

2. Forsgren L, Beghi E, Oun A, Sillanpää M. The epidemiology of epilepsy in Europe-a systematic review. Eur J Neurol. 2005;12:245-53.

3. Rohracher A, Kalss G, Kuchukhidze G, Neuray C, Leitinger M, Höfler J, Kreidenhuber R, Rossini F, Volna K, Mauritz M, Poppert N, Lattanzi S, Brigo F, Trinka E. New anti-seizure medication for elderly epilepsy patients-a critical narrative review. Expert Opin Pharmacother. 2021;22:621-34.

4. Bourdet SV, Gidal BE, Alldredge BK. Pharmacologic management of epilepsy in the elderly. J Am Pharm Assoc (Washington, DC 1996). 2001;41(3):421-36.

5. Trinka E. Epilepsy: comorbidity in the elderly. Acta Neurol Scand Suppl. 2003;180:33-6.

6. Zaccara G, Lattanzi S, Cincotta M, Russo E. Drug treatments in patients with cardiac diseases and epilepsy. Acta Neurol Scand. 2020;142:37-49.

7. Zaccara G, Lattanzi S. Comorbidity between epilepsy and cardiac arrhythmias: implication for treatment. Epilepsy Behav. 2019;97:304-12.

8. Rogawski MA. Revisiting AMPA receptors as an antiepileptic drug target. Epilepsy Curr. 2011;11(56-63):3.

9. Fisher RS, Cross JH, French JA, Higurashi N, Hirsch E, Jansen $\mathrm{FE}$, et al. Operational classification of seizure types by the International League Against Epilepsy: position paper of the ILAE Commission for Classification and Terminology. Epilepsia. 2017;58:522-30.

10. Leppik IE, Brodie MJ, Saetre ER, Rowan AJ, Ramsay RE, Macias F, Jacobs MP. Outcomes research: clinical trials in the elderly. Epilepsy Res. 2006;68(Suppl 1):S71-6.

11. Walker MC, Sander JW. Difficulties in extrapolating from clinical trial data to clinical practice: the case of antiepileptic drugs. Neurology. 1997;49:333-7.

12. Lattanzi S, Trinka E, Del Giovane C, Nardone R, Silvestrini M, Brigo F. Antiepileptic drug monotherapy for epilepsy in the elderly: a systematic review and network meta-analysis. Epilepsia. 2019;60:2245-54.

13. Leppik IE, Wechsler RT, Williams B, Yang H, Zhou S, Laurenza A. Efficacy and safety of perampanel in the subgroup of elderly patients included in the phase III epilepsy clinical trials. Epilepsy Res. 2015;110:216-20.

14. Rohracher A, Zimmermann G, Villanueva V, Garamendi I, Sander JW, Wehner T, Shankar R, Ben-Menachem E, Brodie MJ, Pensel MC, Di Gennaro G, Maurousset A, Strzelczyk A, Rheims S,
Rácz A, Menzler K, Bertol-Alegre V, García-Morales I, LópezGonzález FJ, Toledo M, Carpenter KJ, Trinka E. Perampanel in routine clinical use across Europe: pooled, multicenter, observational data. Epilepsia. 2018;59:1727-39.

15. Liguori C, Manfredi N, Renna R, Izzi F, Pagliuca M, Pagliuca F, Mercuri NB, Fabio P. Comparison of the effectiveness and tolerability of perampanel and brivaracetam: a real-world, observational, retrospective study. Epileptic Disord. 2020;22:309-16.

16. Trinka E, Steinhoff BJ, Nikanorova M, Brodie MJ. Perampanel for focal epilepsy: insights from early clinical experience. Acta Neurol Scand. 2016;133:160-72.

17. Strzelczyk A, Knake S, Kälviäinen R, Santamarina E, Toledo M, Willig S, Rohracher A, Trinka E, Rosenow F. Perampanel for treatment of status epilepticus in Austria, Finland, Germany, and Spain. Acta Neurol Scand. 2019;139:369-76.

18. Brigo F, Lattanzi S, Rohracher A, Russo E, Meletti S, Grillo E, Trinka E. Perampanel in the treatment of status epilepticus: a systematic review of the literature. Epilepsy Behav. 2018;86:179-86.

19. Gidal BE, Laurenza A, Hussein Z, Yang H, Fain R, Edelstein J, Kumar D, Ferry J. Perampanel efficacy and tolerability with enzyme-inducing AEDs in patients with epilepsy. Neurology. 2015;84:1972-80.

20. Villanueva V, Garcés M, López-González FJ, Rodriguez-Osorio X, Toledo M, Salas-Puig J, González-Cuevas M, Campos D, Serratosa JM, González-Giráldez B, Mauri JA, Camacho JL, Suller A, Carreño M, Gómez JB, Montoya J, Rodríguez-Uranga J, SaizDiaz R, de la Aleja JG, Castillo A, López-Trigo J, Poza JJ, Flores J, Querol R, Ojeda J, Giner P, Molins A, Esteve P, Baiges JJ. Safety, efficacy and outcome-related factors of perampanel over 12 months in a real-world setting: the FYDATA study. Epilepsy Res. 2016;126:201-10.

21. Gidal BE, Ferry J, Majid O, Hussein Z. Concentration-effect relationships with perampanel in patients with pharmacoresistant partial-onset seizures. Epilepsia. 2013;54:1490-7.

22. Coccaro EF, Lee R, Vezina P. Cerebrospinal fluid glutamate concentration correlates with impulsive aggression in human subjects. J Psychiatr Res. 2013;47:1247-53.

23. Mula M, Trimble MR, Sander JW. Are psychiatric adverse events of antiepileptic drugs a unique entity? A study on topiramate and levetiracetam. Epilepsia. 2007;48:2322-6.

24. Chung S, Williams B, Dobrinsky C, Patten A, Yang H, Laurenza A. Perampanel with concomitant levetiracetam and topiramate: post hoc analysis of adverse events related to hostility and aggression. Epilepsy Behav. 2017;75:79-85.

25. Meador KJ, Yang H, Piña-Garza JE, Laurenza A, Kumar D, Wesnes KA. Cognitive effects of adjunctive perampanel for partial-onset seizures: a randomized trial. Epilepsia. 2016;57:243-51.

26. Piña-Garza JE, Lagae L, Villanueva V, Renfroe JB, Laurenza A, Williams B, Kumar D, Meador KJ. Long-term effects of adjunctive perampanel on cognition in adolescents with partial seizures. Epilepsy Behav. 2018;83:50-8.

27. Weerdesteyn V, de Niet M, van Duijnhoven HJ, Geurts AC. Falls in individuals with stroke. J Rehabil Res Dev. 2008;45:1195-213.

28. Brigo F, Lattanzi S. Pharmacotherapeutic considerations for lateonset epilepsy. Expert Opin Pharmacother. 2021;22:389-91. 


\section{Authors and Affiliations}

\section{Simona Lattanzi ${ }^{1} @$. Claudia Cagnetti ${ }^{1} \cdot$ Nicoletta Foschi $^{1} \cdot$ Roberta Ciuffini $^{2} \cdot$ Elisa Osanni ${ }^{3} \cdot$ Valentina Chiesa $^{4}$. Filippo Dainese $^{5} \cdot$ Fedele Dono $^{6} \cdot$ Maria Paola Canevini $^{4,7}$. Giacomo Evangelista ${ }^{6}$. Francesco Paladin ${ }^{5}$. Emanuele Bartolini ${ }^{8}$. Federica Ranzato ${ }^{9} \cdot$ Annacarmen Nilo $^{10}$. Giada Pauletto ${ }^{11}$. Daniela Marino ${ }^{12}$. Eleonora Rosati ${ }^{13} \cdot$ Paolo Bonanni $^{3} \cdot$ Alfonso Marrelli $^{14}$}

1 Department of Experimental and Clinical Medicine, Neurological Clinic, Marche Polytechnic University, Via Conca 71, 60020 Ancona, Italy

2 Department of Life, Health and Environmental Sciences, University of L'Aquila, L'Aquila, Italy

3 Epilepsy and Psychopathology Unit, IRCCS Medea, Conegliano, Treviso, Italy

4 Epilepsy Center, San Paolo Hospital, Milan, Italy

5 Epilepsy Center, Neurology Unit, Venice, Italy

6 Department of Neuroscience, Imaging and Clinical Science, “G. D’Annunzio” University of Chieti-Pescara, Chieti, Italy

7 Department of Health Sciences, Università degli Studi, Milan, Italy

8 Neurology Unit, USL Centro Toscana, Prato, Italy

9 Neurology Unit, Epilepsy Center, Vicenza, Italy
10 Clinical Neurology Unit, Department of Neurosciences, S. Maria della Misericordia University Hospital, ASUFC, Udine, Italy

11 Neurology Unit, Department of Neurosciences, S. Maria della Misericordia University Hospital, ASUFC, Udine, Italy

12 Neurology Unit, Department of Cardiac, Thoracic, Neurological and Vascular Sciences, San Donato Hospital, Arezzo, Italy

13 Neurology Unit 2, Neuromuscular and Sense Organs Department, Careggi University Hospital, Florence, Italy

14 Neurophysiopathology Unit, Epilepsy Center, San Salvatore Hospital, L’Aquila, Italy 